\title{
Interplay of spin and orbital magnetogyrotropic photogalvanic effects in InSb/(Al,In)Sb quantum well structures
}

\author{
S. Stachel,${ }^{1}$ P. Olbrich, ${ }^{1}$ C. Zoth, ${ }^{1}$ U. Hagner, ${ }^{1}$ T. Stangl, ${ }^{1}$ C. Karl, ${ }^{1}$ P. Lutz, ${ }^{1}$ V. V. Bel'kov, ${ }^{2}$ S. K. Clowes, ${ }^{3}$ \\ T. Ashley, ${ }^{4}$ A. M. Gilbertson, ${ }^{5}$ and S. D. Ganichev ${ }^{1}$ \\ ${ }^{1}$ Terahertz Center, University of Regensburg, DE-93040 Regensburg, Germany \\ ${ }^{2}$ Ioffe Physical-Technical Institute, Russian Academy of Sciences, 194021 St. Petersburg, Russia \\ ${ }^{3}$ Advanced Technology Institute and SEPNet, University of Surrey, Surrey GU2 7XH, United Kingdom \\ ${ }^{4}$ School of Engineering, University of Warwick, Coventry CV4 7AL, United Kingdom \\ ${ }^{5}$ Blackett Laboratory, Imperial College, London SW7 2BZ, United Kingdom
}

(Received 7 December 2011; revised manuscript received 23 December 2011; published 9 January 2012)

\begin{abstract}
We report on the observation of linear and circular magnetogyrotropic photogalvanic effects in InSb/(Al,In)Sb quantum well structures. We show that intraband (Drude-type) absorption of terahertz radiation in the heterostructures causes a dc electric current in the presence of an in-plane magnetic field. The photocurrent behavior upon variation of the magnetic field strength, temperature, and wavelength is studied. We show that at moderate magnetic fields, the photocurrent exhibits a typical linear field dependence. At high magnetic fields, however, it becomes nonlinear and inverses its sign. The experimental results are analyzed in terms of the microscopic models based on asymmetric relaxation of carriers in the momentum space. We demonstrate that the observed nonlinearity of the photocurrent is caused by the large Zeeman spin splitting in InSb/(Al,In)Sb structures and an interplay of the spin-related and spin-independent roots of the magnetogyrotropic photogalvanic effect.
\end{abstract}

DOI: 10.1103/PhysRevB.85.045305

PACS number(s): 72.40.+w, 73.21.Fg, 72.25.Fe, 72.25.Rb

\section{INTRODUCTION}

Indium-antimonide-based quantum wells (QWs) have attracted growing attention for high-speed transistors, ${ }^{1}$ quantum computing, ${ }^{2,3}$ and infrared lasers. ${ }^{4}$ This novel material is the subject of numerous experimental studies of transport, optical, magneto-optical, and spin-related phenomena. ${ }^{1-14}$ The characteristics driving the interest in this novel narrow gap material are the high carrier mobility, small effective masses, large Landé $g^{*}$ factor, possibility of the mesoscopic spindependent ballistic transport, and a strong spin-orbit coupling. The latter gives rise to a number of optoelectronic effects such as, e.g., terahertz photoconductivity ${ }^{15}$ and the circular photogalvanic effect ${ }^{16-22}$ recently observed in InSb QWs. ${ }^{23}$ Investigation of photogalvanic effects in the presence of a magnetic field should provide further access to nonequilibrium processes in low-dimensional structures, yielding information of such details as the anisotropy of the band spin splitting, processes of momentum and energy relaxation, symmetry properties, and the Zeeman spin splitting (for review, see Refs. 16, 24, and 25).

Here, we report on the observation and detailed study of the magnetogyrotropic photogalvanic effects ${ }^{25,26}$ (MPGE) in $n$-doped InSb/(Al,In)Sb QWs induced by terahertz (THz) radiation. We discuss both the linear magnetogyrotropic photogalvanic effect (LMPGE), which can be induced by linearly polarized or unpolarized radiation, as well as the circular magnetogyrotropic photogalvanic effect (CMPGE), which results in the light helicity-dependent photocurrent and reverses its direction upon switching the sign of the circular polarization. We show that in InSb/(Al,In)Sb QWs, the narrow energy gap and the strong spin-orbit coupling combined with the large Landé $g^{*}$ factor result in a photocurrent orders of magnitude larger than that reported for GaAs- and InAs-based QWs (for review, see Ref. 25). Moreover, in contrast to previous studies, the observed photocurrent exhibits a peculiar magnetic field dependence: while for moderate magnetic fields $(<1 \mathrm{~T})$, the LMPGE current has a typical linear dependence on magnetic field $\boldsymbol{B}$, at higher magnetic fields it becomes nonlinear and reverses its sign. By contrast, the CMPGE remains linear in the whole range of investigated magnetic fields. The experimental results are analyzed in terms of $\operatorname{spin}^{27-29}$ and orbital ${ }^{29-31}$ microscopic models of the magnetogyrotropic photogalvanic effect based on the asymmetry of the relaxation of carriers in the momentum space. We demonstrate that specific magnetic field dependences observed for the LMPGE are due to the nonlinear Zeeman spin splitting in InSb/(Al,In)Sb QWs, which is enhanced by the electron-electron exchange interaction and causes a nonlinear increase of the spin-related MPGE.

\section{SAMPLES AND EXPERIMENTAL TECHNIQUES}

We investigated two $n$-type InSb/(Al,In)Sb single quantum well structures grown by molecular beam epitaxy onto semiinsulating nominally (001)-oriented GaAs substrate. A QW of width $L_{W}$ is confined on each side by an InAlSb barrier, with a Te-modulation-doped layer $20 \mathrm{~nm}$ above the QW (ME1833 and ME2507 with $L_{W}=20$ and $30 \mathrm{~nm}$, respectively). ${ }^{10,13}$ The calculated conduction-band profile, electron wave function, and doping position of the $20-\mathrm{nm} \mathrm{QW}$ are shown in Fig. 1(a). The data are obtained by a self-consistent solution of the Schrödinger and Poisson equations. ${ }^{13}$ The $\mathrm{QW}$ with $L_{W}=20 \mathrm{~nm}(30 \mathrm{~nm})$ width contains a two-dimensional electron gas with the carrier density of $N_{s} \approx 3 \times 10^{11} \mathrm{~cm}^{-2}$ $\left(5 \times 10^{11} \mathrm{~cm}^{-2}\right)$ and the mobility of $\mu_{e} \approx 5 \times 10^{4} \mathrm{~cm}^{2} / \mathrm{V} \mathrm{s}$ $\left(15 \times 10^{4} \mathrm{~cm}^{2} / \mathrm{V} \mathrm{s}\right)$ for $T$ below $77 \mathrm{~K}$. The temperature dependence of $\mu_{e}$ and $N_{s}$ measured in the 20-nm QW structure by low-field Hall effect are shown in Fig. 1(b). The samples have square shape and two pairs of Ohmic contacts on opposite side of the edges (see inset in Fig. 2) oriented along $x \|$ [1 10$]$ and $y \|[110]$. The photocurrents have been investigated in the temperature range of $T=4.2$ to $270 \mathrm{~K}$ using an optical Janis 

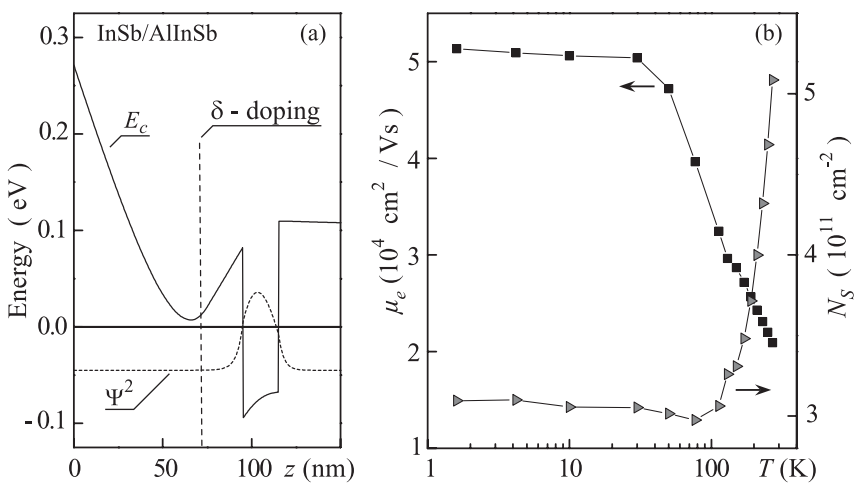

FIG. 1. (a) Conduction-band profile and electron wave function of QW structure with $L_{W}=20 \mathrm{~nm}$ calculated within a self-consistent Schrödinger-Poisson model (Ref. 13). (b) Temperature dependences of mobility $\mu_{e}$ and carrier density $N_{s}$ obtained by the low-field Hall measurements in 20-nm QW sample.

cryostat and a split-coil superconducting magnet. The external magnetic field $\boldsymbol{B}$ up to $\pm 7 \mathrm{~T}$ has been applied parallel to the interface plane along the $x$ direction.

To generate photogalvanic currents, we applied a continuous wave $(\mathrm{cw})$ and pulsed molecular lasers optically pumped by $\mathrm{CO}_{2}$ lasers. For low-power $\mathrm{cw}$ radiation, we used a $\mathrm{CH}_{3} \mathrm{OH}$ laser operating at wavelength $\lambda=118 \mu \mathrm{m}$ (frequency $f=2.5 \mathrm{THz}$ ) with a power $P \approx 2 \mathrm{~mW}$ at the sample position. The radiation was modulated at $120 \mathrm{~Hz}$, allowing the detection of the photoresponse by the standard lock-in technique. Highpower radiation is obtained by a pulsed $\mathrm{NH}_{3}$ laser optically pumped by a transversely excited atmosphere TEA- $\mathrm{CO}_{2}$ laser and operating at wavelengths $\lambda=90.5,148$, or $280 \mu \mathrm{m}$ (frequencies $f=3.3,2$, and $1.1 \mathrm{THz}$, respectively). More details on the system can be found in Refs. 32-36. Here, we used single pulses with a pulse length of about $100 \mathrm{~ns}$, peak power of $P \approx 5 \mathrm{~kW}$, and a repetition rate of $1 \mathrm{~Hz}$. The small

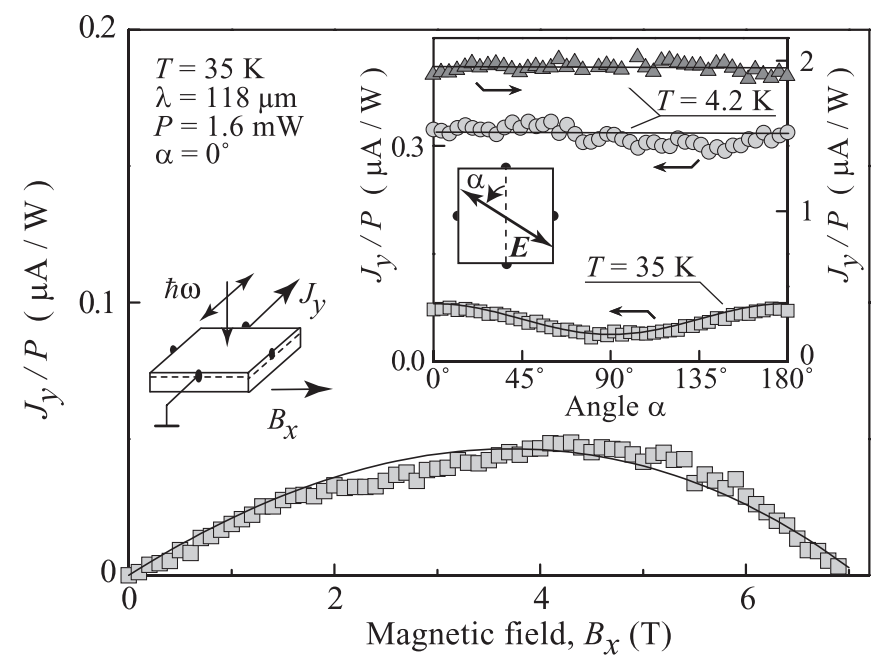

FIG. 2. Magnetic field dependence of $J_{y} / P$ for $\lambda=118 \mu \mathrm{m}$ and $T=35 \mathrm{~K}$. Lines are fit after Eq. (15). The left inset shows the experimental geometry. The right inset shows the photocurrent as a function of the azimuth angle $\alpha$ measured for $T=4.2$ and $35 \mathrm{~K}$ at fixed $B_{x}=+5 \mathrm{~T}$. Triangle symbols correspond to 30-nm, and circle and squared symbols to 20-nm QW structures. duty cycle of about $10^{-7}$ was used in order to avoid the heating of the sample. The photocurrents in unbiased structures are measured via the voltage drop across a $50-\Omega$ load resistor with a storage oscilloscope. The radiation power of $\mathrm{cw}$ and pulsed radiation has been controlled by a pyroelectric detector, calorimeter, and $\mathrm{THz}$ photon drag detector, ${ }^{37}$ respectively. A typical spot diameter is from 1 to $3 \mathrm{~mm}$. The beam has an almost Gaussian form, which is measured by a pyroelectric camera. $^{38}$

All experiments are performed at normal incidence of light. Photocurrents are measured perpendicularly $\left(J_{y}\right)$ and parallel $\left(J_{x}\right)$ to the applied magnetic field $\left(B_{x}\right)$, referred to as transverse and longitudinal photocurrents, respectively. Our lasers emit linearly polarized radiation with the electric field vector of the THz radiation oriented along the $y$ axis. In order to rotate the electric field vector $\boldsymbol{E}$ by the angle $\alpha\left(\alpha=0^{\circ}, \boldsymbol{E} \| y\right)$, we used a $\lambda / 2$ plate. To excite the circular photocurrent, we changed the radiation helicity $P_{\text {circ }}$ by rotating the $\lambda / 4$ plate by the angle $\varphi$ between the initial linear polarization of the laser light and the plate optical axis. In this way, the helicity of the incident light can be varied from -1 (left-handed circular, $\sigma_{-}$) to +1 (right-handed circular, $\sigma_{+}$) according to $P_{\text {circ }}=\sin 2 \varphi$.

\section{RESULTS}

\section{A. Photocurrent induced by linearly polarized and unpolarized radiation}

We shall start by describing the results obtained by irradiating the sample with linearly polarized radiation, which may result only in signals due to the LMPGE and excludes the CMPGE. The magnetic-field-induced photocurrent is studied by applying an in-plane magnetic field $B_{x}$. The observed signal varies with magnetic field strength and its sign depends on the magnetic field direction. While for the 30-nm QW sample no signal is detected at zero magnetic field, in samples with $L_{W}=20 \mathrm{~nm}$ QW we observed a signal at $B_{x}=0$. The origin of this magnetically independent signal ${ }^{39}$ is not within the scope of this paper and will be discussed elsewhere. In the following, we eliminate this contribution by taking $J(|B|)$ as

$$
J_{y}(|B|)=\left[J\left(B_{x}>0\right)-J\left(B_{x}<0\right)\right] / 2
$$

so that only magnetic-field-dependent effects remain.

The transverse photocurrent $J_{y}(|B|)$ excited by the linearly polarized radiation of low-power $\mathrm{cw}$ laser is shown in Fig. 2 as a function of the magnetic field $B_{x}$ and in the right inset in Fig. 2 as a function of the azimuth angle $\alpha$ for a fixed magnetic field $B_{x}=+5 \mathrm{~T}$. While at low temperatures the transverse photocurrent $J_{y}$ comes almost all from the polarization-independent offset, at higher temperature we observed a variation of the photocurrent with rotation of linear polarization $\left[J_{y}=J_{1}+J_{2} \cos (2 \alpha)\right]$. In the longitudinal configuration, we detected only the polarization-dependent photocurrent $J_{x}=J_{3} \sin (2 \alpha)$, which, like the transversal partial current $J_{2} \cos (2 \alpha)$, contributes at higher temperatures only. The experiment reveals that, particularly at low temperatures, the polarization-dependent photocurrent contributions $J_{2} \cos (2 \alpha)$ and $J_{3} \sin (2 \alpha)$ in our samples are substantially smaller than $J_{1}$. Thus, in the following, we focus on the 


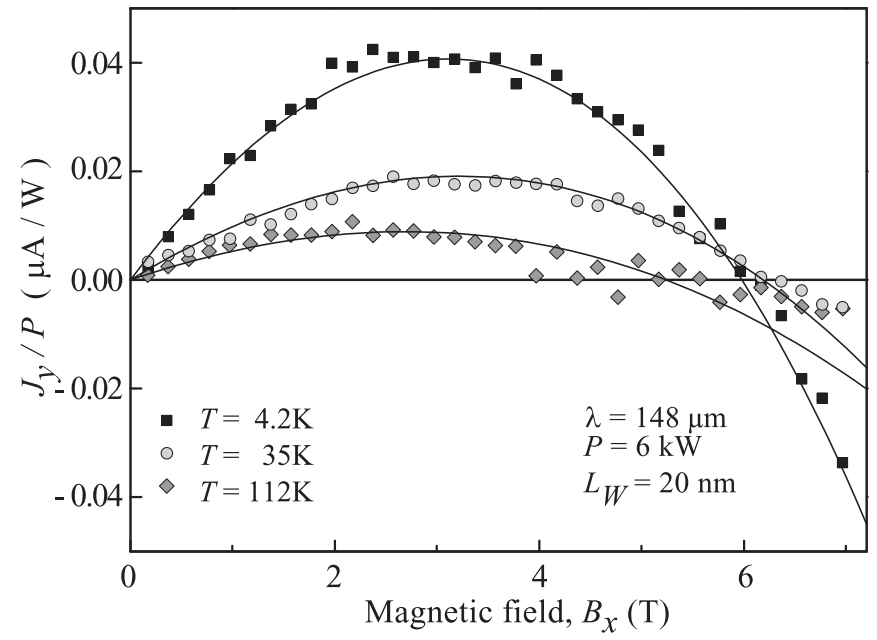

FIG. 3. Magnetic field dependence of $J_{y} / P$ for $\lambda=148 \mu \mathrm{m}$ and different temperatures. Lines are fit after Eq. (15).

polarization-independent photocurrent $J_{y}$ observed in the transverse geometry.

The most striking observation comes from the investigation of the magnetic field dependence of the photocurrent. The general behavior of the photocurrent is that the magnitude of $J_{y}$ is proportional to $B_{x}$ for low magnetic fields only. At higher fields, however, the signal becomes nonlinear: with increasing $B_{x}$, the sign of $d J_{y} / d B_{x}$ changes and, finally, the signal vanishes and for some conditions even reverses its sign. Figure 2 shows such a magnetic field dependence measured applying low-power radiation of the $\mathrm{cw}$ laser with $P \approx 1.6 \mathrm{~mW}$. In Figs. 3 and 4, we plotted the magnetic field dependence of $J_{y}$ excited by the high-power radiation of the pulsed laser. The data obtained for a fixed wavelength of $\lambda=148 \mu \mathrm{m}$ and various temperatures (Fig. 3) and for fixed temperature of $4.2 \mathrm{~K}$ but several wavelengths (Fig. 4). Figure 3 depicts that increasing the temperature reduces the magnitude of the photocurrent, whereas the magnetic field $B_{x} \approx 6.2 \mathrm{~T}$ at which the zero crossing occurs remains almost unchanged. By measuring the temperature dependence for both low-

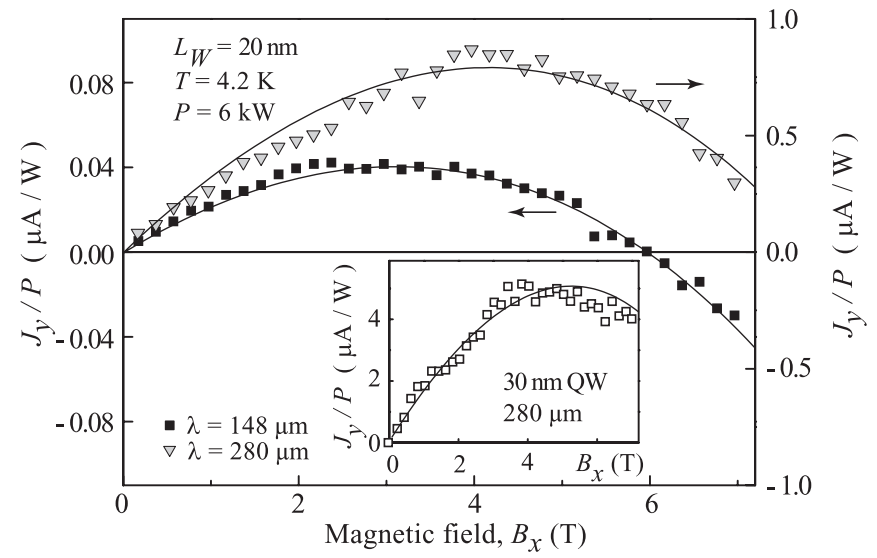

FIG. 4. Dependence of the LMPGE on the magnetic field at $T=$ 4.2 $\mathrm{K}$ for wavelengths of $\lambda=148$ and $280 \mu \mathrm{m}$ obtained for the 20-nm QW structure. The inset shows the LMPGE for the 30-nm QW sample. Lines are fit after Eq. (15).

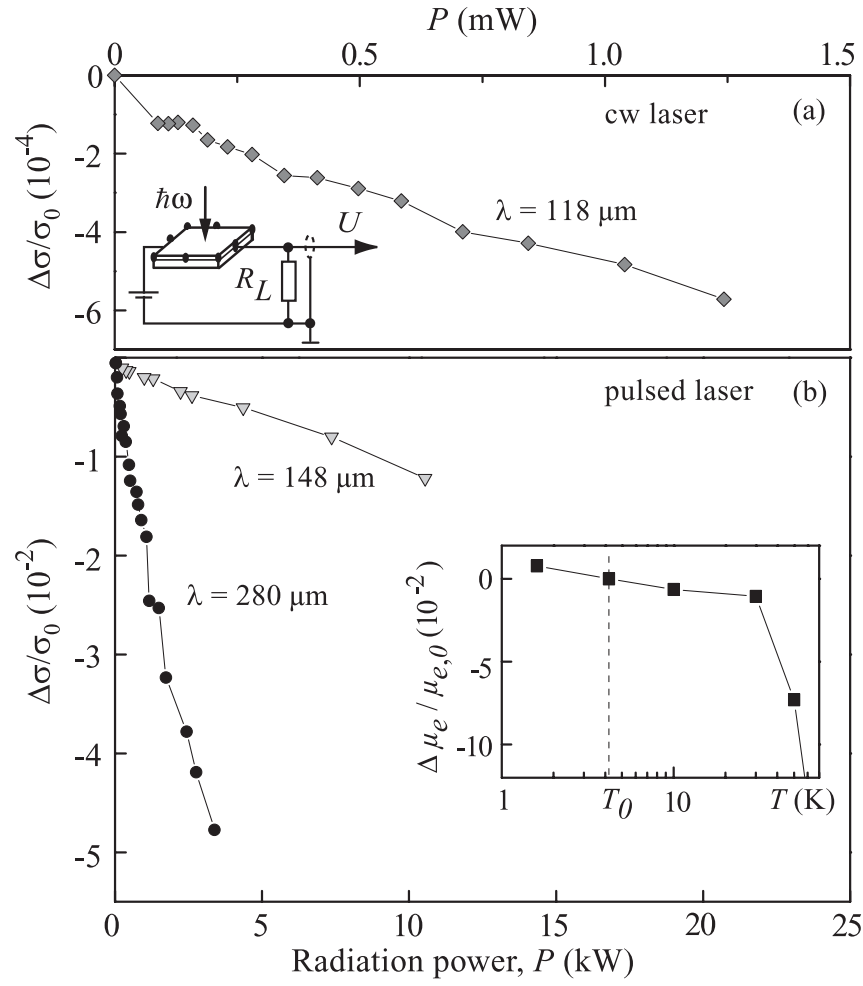

FIG. 5. Relative change in conductivity $\Delta \sigma / \sigma_{0}=\left(\sigma_{i}-\sigma_{0}\right) / \sigma_{0}$ in QW structure with $L_{W}=20 \mathrm{~nm}$ measured versus radiation power $P$ at $T=4.2 \mathrm{~K}$ and $B=0$. The ratio of conductivity under illumination $\sigma_{i}$ and dark conductivity $\sigma_{0}$ is determined from the photoconductive signals measured in the circuit sketched in the inset of the upper plate. (a) Photoconductive signal measured applying cw radiation with wavelength $\lambda=118 \mu \mathrm{m}$. (b) $\Delta \sigma / \sigma_{0}$ measured applying pulsed laser radiation with $\lambda=148$ and $280 \mu \mathrm{m}$. The inset shows a section of the temperature dependence of the relative mobility $\Delta \mu_{e} / \mu_{e, 0}$, where $\mu_{e, 0}$ is the mobility at $T_{0}=4.2 \mathrm{~K}$.

and high-power excitations, we obtained that for $T<8 \mathrm{~K}$ the photocurrent is constant at fixed magnetic field and at higher temperatures rapidly decays showing close to $J \propto 1 / T$ behavior (not shown). In the case of fixed temperature but increasing wavelength (see Fig. 4), the magnitude of the photocurrent increases and the zero crossover is shifted to higher magnetic fields. Finally, we note that sweeping the magnetic field from negative to positive and back, we did not observe a hysteresis.

Our experiments demonstrate that the photocurrent is dominated by a photocurrent contribution, which is insensitive to the radiation polarization. Earlier studies of magnetophotocurrents demonstrated that such polarization-independent photocurrents are caused by the radiation-induced electron gas heating followed by the scattering asymmetry in $\boldsymbol{k}$ space (see Refs. 25, 26, and 28). In order to characterize the electron gas heating in our structures, we investigated the $\mathrm{THz}$ photoconductivity applying the same wavelengths and powers. Figure 5(a) shows the photoconductive signal excited by the $\mathrm{cw} \mathrm{THz}$ laser as a function of radiation power. The observed decrease of the structure conductivity with increasing $\mathrm{THz}$ radiation (negative photoconductivity) provides the evidence for the electron gas heating. Indeed, Hall measurements [see Fig. 1(b) and the inset in Fig. 5(b)] show that a rise of 
temperature results in the decrease of mobility and, consequently, in the lowering of conductivity. The data for pulsed excitation, presented in Fig. 5(b), demonstrate that an increase of the radiation power by about six orders of magnitude results in a change of the relative photoconductivity $\left|\Delta \sigma / \sigma_{0}\right|$ by two orders of magnitude. We attribute the observed nonlinearity of the photoconductive response to nonlinear energy losses in InSb QWs at low temperature, which, consequently, cause a strongly nonlinear dependence of the electron temperature on the absorbed energy. ${ }^{24}$ A comparison of the data obtained at $\lambda=148$ and $280 \mu \mathrm{m}$ demonstrates essentially stronger electron gas heating at a longer wavelength. This observation is in a good agreement with the frequency dependence of the Drude-type absorption. Figure 5(b) shows that for radiation power of several kilowatts, relative photoconductivity achieves values as high as $10^{-2}$ to $10^{-1}$. Comparison of these values with the mobility data [see the inset in Fig. 5(b)] shows that pulsed $\mathrm{THz}$ radiation used here can heat up the electron gas by tens kelvin. ${ }^{40}$

\section{B. Photocurrent induced by circularly polarized radiation}

We will now describe the results for irradiation with circularly (elliptically) polarized light, which is obtained using a $\lambda / 4$ plate. The ellipses on top of Fig. 6 illustrate the polarization states for various angles $\varphi$. The resulting polarization state is given by the Stokes parameters ${ }^{42} S_{1}=\cos ^{2}(2 \varphi)$ and $S_{2}=\sin (4 \varphi) / 2$, describing the degree of linear polarization, and $S_{3} \equiv P_{\text {circ }}$. The photocurrent detected in the transverse geometry is well described by $J_{y}=J_{1}+\left(J_{2} / 2\right) \cos (4 \varphi)$. The photocurrent consists of polarization-independent contribution $J_{1}$ and a contribution that is proportional to the degree of linear polarization, i.e., just the same as discussed in the previous section. In the longitudinal geometry $\left(J_{x} \| B_{x}\right)$, however, we observed a new contribution to the photocurrent. It manifests itself in the helicity dependence of the signal. The dependence of the photocurrent $J_{x}$ on $\varphi$ is shown in Fig. 6. It is well described by $J_{x}(\varphi)=\left(J_{3} / 2\right) \sin (4 \varphi)+J_{C} \sin (2 \varphi)+\xi$. Here,

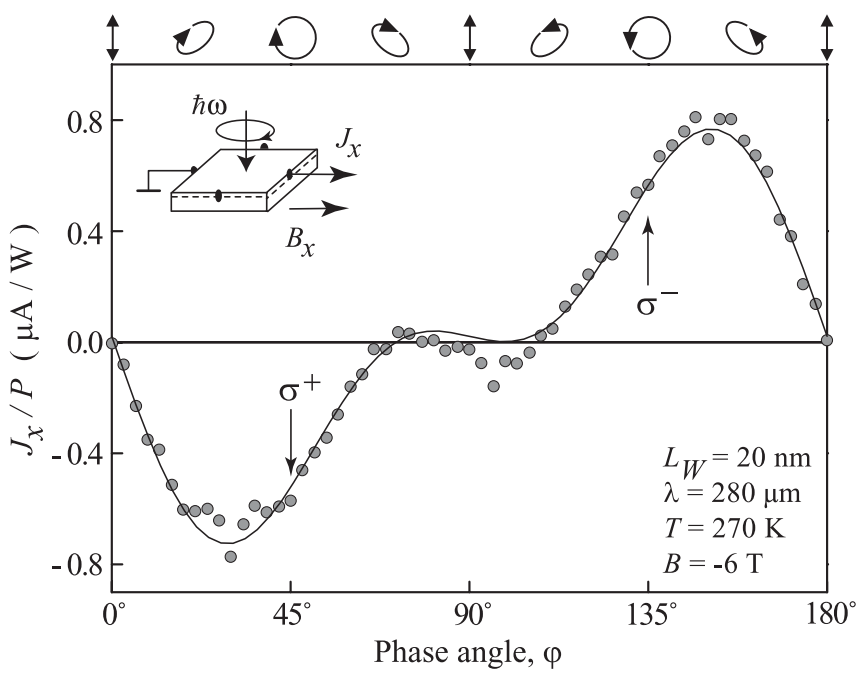

FIG. 6. Helicity dependence of the photocurrent $J_{x}$ measured for $B_{x}=-6 \mathrm{~T}$ and $\lambda=280 \mu \mathrm{m}$ with subtracted offset $\xi$. The inset shows the experimental geometry. The ellipses on top illustrate the polarization states for various $\varphi$.

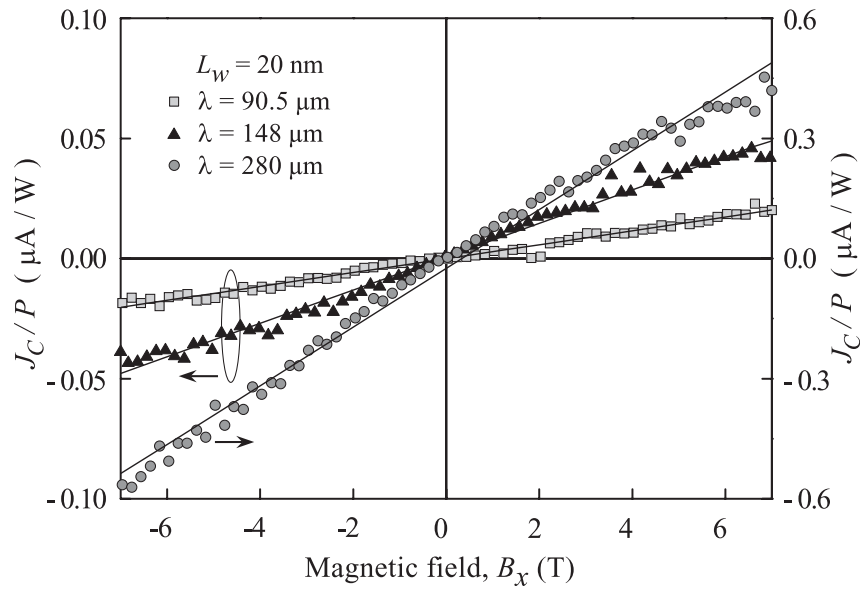

FIG. 7. Magnetic field dependence of $J_{x} / P$ for wavelengths of $\lambda=90.5,148$, and $280 \mu \mathrm{m}$ at $T=270 \mathrm{~K}$.

the first term is again just the contribution proportional to $J_{3}$ in the described above experiments with linearly polarized radiation. It reflects the degree of linear polarization and vanishes for circularly polarized light. The second term is proportional to the radiation helicity $P_{\text {circ }}$. This circular photocurrent changes its sign by switching the light helicity from -1 to +1 . Note that the observed offset $\xi$ is much smaller than $J_{3}$ and $J_{C}$ and is subtracted from the data of Fig. 6. We will focus on circular photocurrent in the longitudinal geometry, thus, we can extinguish all other possible effects by

$$
J_{C}=\left[J_{x}\left(\sigma^{+}\right)-J_{x}\left(\sigma^{-}\right)\right] / 2 .
$$

Figure 7 shows the magnetic field dependence of the circular photocurrent $J_{C}$ measured in the $20-\mathrm{nm}$ QW structure for different wavelengths. Similarly to the photocurrent induced by linearly polarized radiation, its magnitude normalized by the radiation power substantially increases for longer wavelengths, a fact which can also naturally be attributed to the increase of the Drude absorption. However, unlike the photocurrent induced by linearly polarized radiation (Figs. 2-4), the circular photocurrent remains proportional to the magnetic field $B_{x}$ up to the highest field applied, $|B|=7 \mathrm{~T}$. The same behavior has been observed in the 30-nm structure (not shown).

\section{DISCUSSION}

All our observations at low magnetic field exhibit the recognized MPGE behavior, which by definition is a magneticfield-induced photocurrent related to the gyrotropic symmetry of the system. ${ }^{26}$ In particular, the observed linear coupling to the magnetic field, the in-plane anisotropy of the photocurrent, as well as the polarization dependences all follow the symmetry arguments for the MPGE. The current perpendicular to the magnetic field is dominated by the polarization-independent contribution (LMPGE) and is therefore driven by relaxation processes. ${ }^{28,41}$ At the same time, the longitudinal magnetic-field-induced photocurrent excited by circularly polarized radiation is solely governed by the photon angular momentum (CMPGE) ${ }^{27}$ Comparison of our data on the magnetic-field-induced photocurrents with that reported earlier for GaAs- and InAs-based QW structures 
(for review, see Ref. 25) shows that it is much stronger in InSb QWs by at least two orders of magnitude. We note that for the 30-nm QWs compared to our 20-nm QW, we detected 10 times larger photoresponses (see the inset in Fig. 2 and the data for $\lambda=280 \mu \mathrm{m}$ in Fig. 4). While the general features of our signals are in agreement with previous results for III-V QWs, the magnetic field dependences of the LMPGE and the CMPGE in InSb-based QWs have a contradictory behavior: the LMPGE is nonlinear and the CMPGE is linear. We will now discuss separately the LMPGE and CMPGE in terms of the interplay between the spin and orbital (nonspin) related relaxation processes. We will show that this interplay results in the surprising magnetic field behavior.

\section{A. Linear MPGE}

The spin-related origin of the LMPGE is a consequence of the electron gas heating followed by spin-dependent scattering. ${ }^{25,28,41}$ The latter is due to the spin-orbit interaction in gyrotropic media, such as InSb- and GaAs-based low-dimensional structures, which yields a scattering matrix element being proportional to $\left[\boldsymbol{\sigma} \times\left(\boldsymbol{k}+\boldsymbol{k}^{\prime}\right)\right]$. Here, $\boldsymbol{k}$ and $\boldsymbol{k}^{\prime}$ are the initial and the scattered wave vectors and $\sigma$ is the vector composed of the Pauli matrices, and only structural inversion asymmetry is assumed. This spin-dependent scattering results in an asymmetric relaxation of the hot electrons shown by the different thickness of the arrows in Fig. 8(a) and causes oppositely directed electron fluxes $\boldsymbol{i}_{ \pm 1 / 2}$ in the spin subbands. Consequently, a spin current, defined as the difference between the fluxes, is given by $\boldsymbol{J}_{s}=1 / 2\left(\boldsymbol{i}_{+1 / 2}-\boldsymbol{i}_{-1 / 2}\right)$. At nonzero magnetic field, e.g., $B_{x}$, the Zeeman effect causes an equilibrium spin polarization parallel to the magnetic field and the fluxes become unbalanced due to the unequal equilibrium population of the spin subbands. The average electron spin $s$ is equal to

$$
s=\frac{1}{2} \frac{N_{+1 / 2}-N_{-1 / 2}}{N_{+1 / 2}+N_{-1 / 2}} .
$$

Such an imbalance results in a net electric current $\boldsymbol{j}_{\text {spin }}$ given by the sum of the fluxes $\boldsymbol{j}_{\text {spin }}=-e\left(\boldsymbol{i}_{+1 / 2}+\boldsymbol{i}_{-1 / 2}\right)$, where $-e$ is the electron charge. Assuming that the fluxes $\boldsymbol{i}_{ \pm 1 / 2}$ are proportional to the carrier densities in the spin subbands $N_{ \pm 1 / 2}$, one obtains

$$
\boldsymbol{j}_{\text {spin }}=-4 e s \boldsymbol{J}_{s} .
$$

We note that while in the theoretical consideration the current density $\boldsymbol{j}$ is used, in the experiments, the electric current $\boldsymbol{J}$ is measured, which is proportional to the current density $\boldsymbol{j}$.
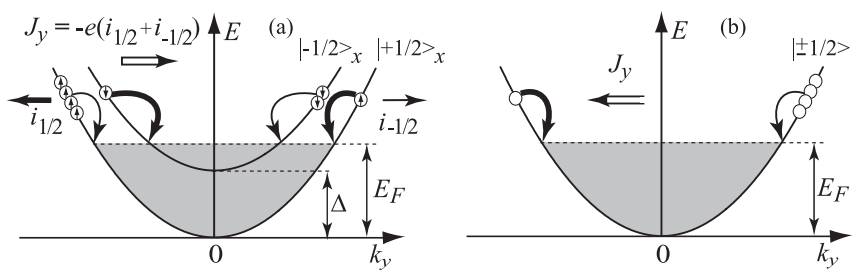

FIG. 8. Models of magnetogyrotropic photogalvanic currents: (a) spin-dependent LMPGE; (b) orbital LMPGE.
At low magnetic fields with the Fermi energy $E_{F}$ larger than the energy of the Zeeman spin splitting, $s$ is a linear function of magnetic field $\boldsymbol{B}$ and is given by

$$
\boldsymbol{s}=-\frac{\Delta}{4 E_{F}} \frac{\boldsymbol{B}}{B},
$$

where $\Delta=g^{*} \mu_{B} B$ is the energy of the Zeeman spin splitting and $\mu_{B}$ is the Bohr magneton. However, in the high-field limit for $|\Delta|>2 E_{F}$, one of the spin subbands will be completely depopulated. Obviously, in this case the average spin $s=$ $\pm 1 / 2$ and $J_{y}$ saturates.

The behavior of the spin-dependent LMPGE, $J_{y} \propto s$, over all magnetic fields can be obtained taking into account that in thermal equilibrium, the densities $N_{ \pm 1 / 2}$ are determined by

$$
N_{ \pm 1 / 2} \propto \sum_{\boldsymbol{k}}\left[\exp \left(\frac{\varepsilon_{\boldsymbol{k}} \pm \Delta / 2-\mu}{k_{B} T_{e}}\right)+1\right]^{-1},
$$

where $\varepsilon_{\boldsymbol{k}}=\hbar^{2} k^{2} /\left(2 m^{*}\right)$ is the kinetic energy, $m^{*}$ is the effective mass, $\mu$ is the chemical potential, $k_{B}$ is the Boltzmann constant, and $T_{e}$ is the electron temperature. Effects on $N_{ \pm 1 / 2}$ due to nonparabolicity of the subbands ${ }^{3}$ will be weak compared to the Boltzmann redistribution from the Zeeman spin splitting, and are therefore ignored. Straightforward summation over the wave vector $\boldsymbol{k}$ yields

$$
s=\frac{1}{2} \frac{\ln \left\{\left[1+\exp \left(\frac{\mu-\Delta / 2}{k_{B} T_{e}}\right)\right] /\left[1+\exp \left(\frac{\mu+\Delta / 2}{k_{B} T_{e}}\right)\right]\right\}}{\ln \left\{\left[1+\exp \left(\frac{\mu-\Delta / 2}{k_{B} T_{e}}\right)\right] \times\left[1+\exp \left(\frac{\mu+\Delta / 2}{k_{B} T_{e}}\right)\right]\right\}} .
$$

Equation (7) describes the average spin of two-dimensional carriers in an external magnetic field for a fixed chemical potential $\mu$. If, instead, the carrier density $N_{s}=N_{+1 / 2}+$ $N_{-1 / 2}$ like in our case is fixed, Eq. (7) should be supplemented with the following equation for the chemical potential:

$$
\begin{aligned}
\mu= & k_{B} T_{e} \ln \left[\sqrt{\exp \left(\frac{2 \pi N_{s} \hbar^{2}}{m^{*} k_{B} T_{e}}\right)+\cosh ^{2}\left(\frac{\Delta}{2 k_{B} T_{e}}\right)-1}\right. \\
& \left.-\cosh \left(\frac{\Delta}{2 k_{B} T_{e}}\right)\right] .
\end{aligned}
$$

The magnetic field dependence of $s$ given by Eq. (7) is nonlinear, saturating at $|s|=1 / 2$. However, the deviation from linear dependence for the degenerate electron gas occurs at rather high magnetic fields when the average spin projection is close to $\pm 1 / 2$. Therefore, we suggest that other effects resulting in a nonlinear magnetic field dependence of the electron spin are responsible for the observed reversal of the electric current with the field increase. As a possible origin of this effect, we consider exchange interaction between electrons, which is known to lead to a nonlinearity of the Zeeman splitting on the external magnetic field at moderate fields. ${ }^{11,14}$ In this case, the effective $g^{*}$ factor besides $g_{0}$, the Landé factor like at $B=0$, contains a contribution linear in the spin polarization

$$
g^{*}=g_{0}+2|s| g^{* *}, \quad \Delta=\left(g_{0}+2|s| g^{* *}\right) \mu_{B} B,
$$

where $2|s| g^{* *}$ is the contribution to the $g^{*}$ factor caused by the exchange interaction. Equations (7) and (9) supplement each other and are to be solved together. The calculated magnetic 


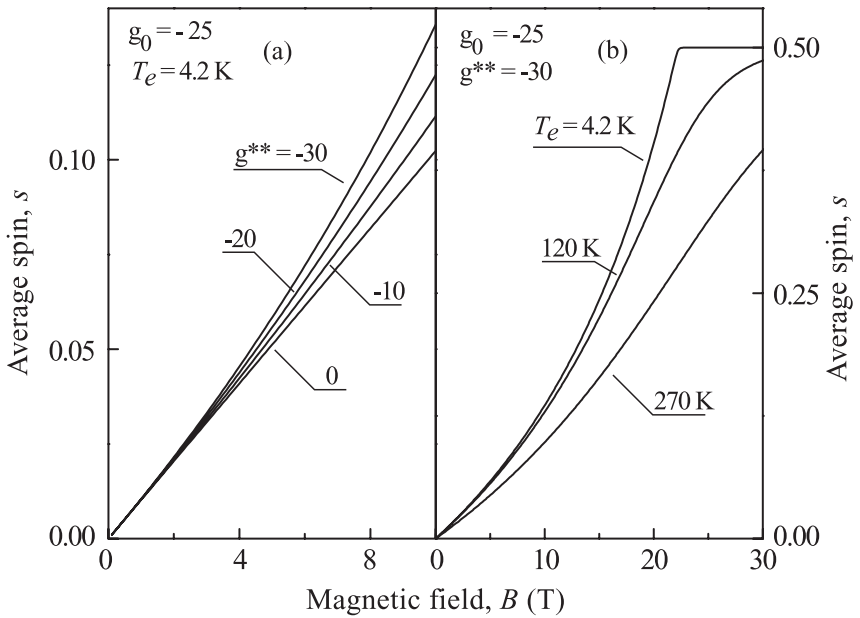

FIG. 9. Average spin in 20-nm QW structures obtained by selfconsistent calculations of Eqs. (7) and (9) as a function of the magnetic field. For calculation, we used $g_{0}=-25$ and an effective mass $m^{*}=$ $0.02 m_{0}$. Average spin calculated for (a) fixed temperature but for various values of the exchange interaction given by the parameter $g^{* *}$ indicated by numbers next to the curves; (b) fixed exchange interaction $g^{* *}=-30$ but various electron temperatures $T_{e}$.

field behavior of the average spin and, consequently, the photocurrent $\left(J_{y} \propto s\right)$, is plotted in Fig. 9. For calculation, we used $g_{0}=-25$ and an effective mass $m^{*}=0.02 m_{0}$ determined by magnetotransport experiments ${ }^{11}$ and cyclotron resonance data (not shown), respectively. Figure 9(a) shows the average spin calculated for $20-\mathrm{nm}$ QW structures at fixed temperature but for various values of the exchange interaction given by the parameter $g^{* *}$. At low temperatures, for $g^{* *}=0$ and $|\Delta| \leqslant 2 E_{F}$, we obtain a linear dependence of the average electron spin on the Zeeman splitting following the well-known behavior described by Eq. (5). The exchange interaction results in a superlinear magnetic field dependence of $s(B)$ so that for $g^{* *}=-30$ the average spin is substantially enhanced already at a magnetic field of several Tesla. Finally, for $|\Delta|>2 E_{F}$, one of the spin subbands will be completely depopulated and $|s|=1 / 2$. Note that $g^{* *}=-30$ is obtained in InSb QWs similar to our structures by magnetotransport measurements. ${ }^{11}$ Using this value, we calculated how electron temperature influences the average spin. The results are plotted in Fig. 9(b). The data show that an increase of the temperature results in a decrease of spin polarization and reduces the nonlinearity. However, for magnetic fields below $7 \mathrm{~T}$ and temperatures below $\approx 130 \mathrm{~K}$, used in experiments here, $s$ remains nearly unchanged by the temperature.

While spin-mediated relaxation can produce a nonlinear signal, it can not cause the observed sign reversal of the photocurrent. Thus, we consider another known mechanism of the LMPGE based on an asymmetric relaxation due to the Lorentz force acting on heated carriers, ${ }^{29-31}$ which may provide an additional contribution to the total photocurrent. The effect is illustrated in Fig. 8(b). Similar to the spin-related MPGE, the current stems from the asymmetric energy relaxation of the hot electrons. Now, however, this asymmetry is caused by the scattering correction being linear in the wave vector $\boldsymbol{k}$ and in the magnetic field $\boldsymbol{B}$, which is allowed in gyrotropic media only. ${ }^{29-31}$ Microscopically, this term is caused by structural inversion asymmetry (SIA) and/or bulk inversion asymmetry (BIA). This process, however, is independent of the spin and the corresponding scattering rate, e.g., SIA, is given by

$$
\boldsymbol{W}_{\boldsymbol{k} \boldsymbol{k}^{\prime}}=W_{0}+w_{\mathrm{SIA}}\left[\boldsymbol{B} \times\left(\boldsymbol{k}+\boldsymbol{k}^{\prime}\right)\right]_{z},
$$

where $W_{0}$ is the field-independent term and $w_{\text {SIA }}$ is a measure of the structure inversion asymmetry. Due to magnetic-fielddependent scattering, transitions to positive and negative $k_{y}^{\prime}$ states occur with different probabilities. Therefore, hot electrons with opposite $k_{y}$ have different relaxation rates in the two spin subbands. In Fig. 8(b), this difference is indicated by arrows of different thicknesses. The resulting electric current is given by

$$
j_{\text {orb }}=-2 e \sum_{k} v_{k} f_{k},
$$

where $\boldsymbol{v}_{\boldsymbol{k}}=\hbar \boldsymbol{k} / \mathrm{m}^{*}$ is the electron velocity and $f_{\boldsymbol{k}}$ is the electron distribution function. The latter is found from the Boltzmann equation

$$
G_{k}-\sum_{\boldsymbol{k}^{\prime}}\left[\boldsymbol{W}_{\boldsymbol{k} \boldsymbol{k}^{\prime}} f_{\boldsymbol{k}^{\prime}}\left(1-f_{\boldsymbol{k}}\right)-W_{\boldsymbol{k}^{\prime} \boldsymbol{k}} f_{\boldsymbol{k}}\left(1-f_{\boldsymbol{k}^{\prime}}\right)\right]=0,
$$

where the generation term $G_{\boldsymbol{k}}$ describes electron gas heating by radiation. Since the scattering rate (10) contains the asymmetric part proportional to $w_{\mathrm{SIA}} B$, the asymmetric part of the distribution function $f_{k}$ and, consequently, the photocurrent $\boldsymbol{j}_{\text {orb }}$ is linearly coupled with the magnetic field and the degree of SIA

$$
j_{\text {orb }} \propto w_{\text {SIA }} B .
$$

We note that this dependence remains linear in the magnetic fields up to ${ }^{30,31}$

$$
B \approx \frac{\pi^{2} \hbar c}{e L_{W}^{2}},
$$

which for $L_{W} \approx 20 \mathrm{~nm}$ is about $25 \mathrm{~T}$, i.e., much larger than fields used in our experiment. Here, $e$ is electron charge and $c$ is the speed of light.

On the phenomenological level, both mechanisms are described by the same equations ${ }^{29-31}$ and the total current is given by the sum of their contributions

$$
j_{y}=j_{\text {spin }}+j_{\text {orb }} .
$$

Taking into account only the dependence on the magnetic field given by Eqs. (4) and (13), we used for the fitting curves $j_{\text {spin }}=a \cdot s(B)$ and $j_{\text {orb }}=b \cdot B$, where $a$ and $b$ are fitting parameters. The phenomenological similarity hinders the decomposition of both terms because the spin contribution $J_{\text {spin }} \propto s$ and the orbital one $J_{\text {orb }} \propto B$ behave identically under a variation of the radiation's polarization state and the orientation of the magnetic field relative to the crystallographic axes. Our above consideration shows, however, that the behavior of the photocurrent upon a variation of the magnetic field strength is different for these two mechanisms. Combining spin and nonspin mechanisms and assuming they have opposite signs, we can explain the nonlinear magnetic field behavior, in particular, the reversal of the photocurrent direction. Figure 2 shows the results of calculations fitted to the 
experimental data obtained at low-power excitation, which just slightly increase the electron temperature $T_{e}$ above the lattice temperature $T$ [see Fig. 5(a)]. Using the lattice temperature for calculations and scaling $J_{\text {spin }}$ and $J_{\text {orb }}$ magnitudes, we obtained a good agreement between experiment and the theory in the whole magnetic field range. Figures 3 and 4 demonstrate that Eq. (15) also describes well the data for the high-power excitation where the electron temperature is by tens of degrees larger than the lattice one [see Fig. 5(b)]. As discussed above, the fact that in these experiments magnetic fields below $7 \mathrm{~T}$ and temperatures below $120 \mathrm{~K}$ are used, the dependence due to the Zeeman splitting is very weak (see Fig. 9). Therefore, we obtain good agreement for both lattice temperatures and electron temperatures assumed to exceed the lattice temperature by several tens of degrees.

The calculations show that these mechanisms yield photocurrents of comparable strength. At low magnetic fields, the total current is dominated by the orbital mechanism. However, even at moderate magnetic fields, the nonlinear increase of the average spin due to the exchange interaction causes an enhancement of the spin-related LMPGE, which at high fields becomes the major origin. The fact that the orbital effect provides a comparable contribution to the spin-related effect is surprising, particularly when taking into account that InSb QWs are characterized by the strong spin-orbit coupling and enhanced magnetic properties. Orbital effects, however, are also enhanced in InSb QWs. The reason is the narrow gap leading to a small effective mass of electrons. As demonstrated in Refs. 30 and 31, the orbital current increases with a lower effective mass.

\section{B. Circular MPGE}

The signature of the CPMGE is that the signal is proportional to the radiation helicity and, consequently, reverses the sign upon switching the helicity from left to right circular polarization (see Fig. 6). In a similar approach to the LMPGE, we consider the interplay between the spin and nonspin mechanisms. We first discuss the spin-related contribution, which is microscopically due to the spin galvanic effect. ${ }^{27}$ For the geometry shown in the inset of Fig. 6, the magnetic field dependence of the CMPGE photocurrent caused by the spin galvanic effect (see Fig. 10) is given by ${ }^{27}$

$$
J_{x} \propto-\frac{\omega_{L} \tau_{s \perp}}{1+\left(\omega_{L} \tau_{s}\right)^{2}} S_{0 z}
$$

where $\tau_{s}=\sqrt{\tau_{s \|} \tau_{s \perp}}$ and $\tau_{s \|}, \tau_{s \perp}$ are the longitudinal and transverse electron spin relaxation times, the Larmor frequency is given by $\omega_{L}=g^{*} \mu_{\mathrm{B}} B_{x} / \hbar$, and $S_{0 z}=\tau_{s \|} \dot{S}_{z}$ is the steadystate electron spin polarization in the absence of a magnetic field. It is seen that the photocurrent should follow the Hanle law: it achieves the maximum of an in-plane spin and consequently the current at $\omega_{L} \tau_{s}$ about unity and vanishes for higher magnetic fields. The spin relaxation time in our 20-nm InSb-based QW has been studied applying the circularly polarized pump-probe technique, yielding for liquid-helium temperature $\tau_{s} \approx 0.1$ ps and $g^{*}=-45$ (see Ref. 13). Thus, the photocurrent maximum is expected for magnetic fields about 2.5 T. In our experiments, however, the current linearly rises with the magnetic field and does not exhibit any nonlinearities.

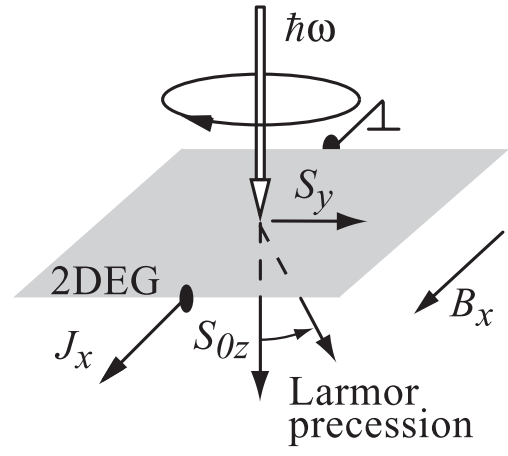

FIG. 10. Model for the spin-related CMPGE. The excitation with circularly polarized light yields a spin orientation $S_{0 z}$. An in-plane component $S_{y}$ of the nonequilibrium spin is generated by the Larmor precession.

This fact forces a conclusion that the spin-galvanic effect does not contribute to the CMPGE.

Microscopically, the orbital contribution to the CMPGE appears similarly to that of the LMPGE current described above. $^{30,31}$ The current is caused by the action of the Lorentz force on the orbital motion of the two-dimensional electrons in the radiation field. Under irradiation with circularly polarized light, electrons perform a cyclic motion. In the system with SIA/BIA, the presence of an in-plane magnetic field pointed along the [110] or [1피 axes forces them to flow predominantly along the direction of $\boldsymbol{B}$. Note that the circular photocurrent, sensitive to the radiation helicity sign, is generated due to a retardation between the rotating electric field of the radiation and the electron velocity. Therefore, it reaches a maximum at $\omega \tau \approx 1$ (here, $\omega=2 \pi f$ is the radiation angular frequency and $\tau$ is the scattering time) and vanishes for much lower or higher frequencies. The microscopic theory of this effect is given in Refs. 30 and 31. Like the orbital LMPGE, the resulting orbital current $J_{C}$ is caused by the $B$-dependent corrections to the scattering probability, Eq. (10). For QWs with $L_{W}=20 \mathrm{~nm}$, it is linearly coupled with magnetic field up to $B$ about $25 \mathrm{~T}$ [see Eq. (14)]. Thus, on the basis of the magnetic field behavior, we conclude that the CMPGE in InSb-based QWs is dominated by the orbital mechanism, which is in this material enhanced due to the small energy band gap.

\section{SUMMARY}

Summarizing, our experiments of $\mathrm{THz}$ radiation-induced linear and circular MPGE in InSb-based QW structures show that due to the narrow energy gap, strong magnetic property, and strong spin-orbit coupling, the effect is substantially enhanced compared to other III-V materials. The measurements demonstrate that both spin and orbital mechanisms of the MPGE contribute to the signal, yielding the current contributions of comparable strength. The observed strong nonlinear behavior of the LMPGE is caused by the nonlinearity of the Zeeman spin splitting and supports recent conclusions on the high polarization-dependent spin susceptibility of a two-dimensional electron gas in InSb-based QWs being much larger than observed in larger mass systems. ${ }^{43}$ 


\section{ACKNOWLEDGMENTS}

We thank S. A. Tarasenko, L. E. Golub, and V. Lechner for fruitful discussions. Support from DFG (SFB 689), Linkage
Grant of IB of BMBF at DLR, and RFBR is acknowledged. S.K.C. gratefully acknowledges support by EPSRC-UK under Grant No. EP/E055583-1 and A.M.G. the support of EPSRC under Grant No. EP/F065922/1.
${ }^{1}$ T. Ashley, L. Buckle, S. Datta, M. T. Emeny, D. G. Hayes, K. P. Hilton, R. Jefferies, T. Martin, T. Phillips, D. J. Waliis, P. J. Wilding, and R. Chan, Electron. Lett. 43, 14777 (2007).

${ }^{2}$ G. A. Khodaparast, R. E. Doezema, S. J. Chung, K. J. Goldammer, and M. B. Santos, Phys. Rev. B 70, 155322 (2004).

${ }^{3}$ K. L. Litvinenko, B. N. Murdin, J. Allam, C. R. Pidgeon, M. Bird, K. Morris, W. Branford, S. K. Clowes, L. F. Cohen, T. Ashley, and L. Buckle, New J. Phys. 8, 49 (2006).

${ }^{4}$ A. D. Andreev, E. P. O'Reilly, A. R. Adams, and T. Ashley, Appl. Phys. Lett. 78, 2640 (2001).

${ }^{5}$ H. Chen, J. J. Heremans, J. A. Peters, N. Goel, S. J. Chung, and M. B. Santos, Appl. Phys. Lett. 84, 5380 (2004).

${ }^{6}$ N. Goel, J. Graham, J. C. Keay, K. Suzuki, S. Miyashita, M. B. Santos, and Y. Hirayama, Phys. E (Amsterdam) 26, 455 (2005).

${ }^{7}$ H. Chen, J. J. Heremans, J. A. Peters, N. Goel, S. J. Chung, and M. B. Santos, Appl. Phys. Lett. 86, 032113 (2005).

${ }^{8}$ J. M. S. Orr, P. D. Buckle, M. Fearn, C. J. Storey, L. Buckle, and T. Ashley, New J. Phys. 9, 261 (2007).

${ }^{9}$ J. M. S. Orr, A. M. Gilbertson, M. Fearn, O. W. Croad, C. J. Storey, L. Buckle, M. T. Emeny, P. D. Buckle, and T. Ashley, Phys. Rev. B 77, 165334 (2008).

${ }^{10}$ A. M. Gilbertson, W. R. Branford, M. Fearn, L. Buckle, P. D. Buckle, T. Ashley, and L. F. Cohen, Phys. Rev. B 79, 235333 (2009)

${ }^{11}$ B. Nedniyom, R. J. Nicholas, M. T. Emeny, L. Buckle, A. M. Gilbertson, P. D. Buckle, and T. Ashley, Phys. Rev. B 80, 125328 (2009)

${ }^{12}$ O. J. Pooley, A. M. Gilbertson, P. D. Buckle, R. S. Hall, L. Buckle, M. T. Emeny, M. Fearn, L. F. Cohen, and T. Ashley, New J. Phys. 12, 053022 (2010).

${ }^{13}$ M. A. Leontiadou, K. L. Litvinenko, A. M. Gilbertson, C. R. Pidgeon, W. R. Branford, L. F. Cohen, M. Fearn, T. Ashley, M. T. Emeny, B. N. Murdin, and S. K. Clowes, J. Phys.: Condens. Matter 23, 035801 (2011).

${ }^{14}$ K. F. Yang, H. W. Liu, T. D. Mishima, M. B. Santos, K. Nagase, and Y. Hirayama, New J. Phys. 13, 083010 (2011).

${ }^{15}$ F. Gouider, Yu. B. Vasilyev, M. Bugár, J. Könemann, P. D. Buckle, and G. Nachtwei, Phys. Rev. B 81, 155304 (2010).

${ }^{16}$ E. L. Ivchenko and S. D. Ganichev, in Spin Photogalvanics in Spin Physics in Semiconductors, edited by M. I. D’yakonov (Springer, Berlin, 2008).

${ }^{17}$ S. D. Ganichev, E. L. Ivchenko, and W. Prettl, Phys. E (Amsterdam) 14, 166 (2002).

${ }^{18}$ S. D. Ganichev, V. V. Bel'kov, P. Schneider, E. L. Ivchenko, S. A. Tarasenko, W. Wegscheider, D. Weiss, D. Schuh, E. V. Beregulin, and W. Prettl, Phys. Rev. B 68, 035319 (2003).

${ }^{19}$ S. D. Ganichev, Petra Schneider, V. V. Bel'kov, E. L. Ivchenko, S. A. Tarasenko, W. Wegscheider, D. Weiss, D. Schuh, B. N. Murdin, P. J. Phillips, C. R. Pidgeon, D. G. Clarke, M. Merrick, P. Murzyn, E. V. Beregulin, and W. Prettl, Phys. Rev. B 68, 081302 (2003).

${ }^{20}$ M. Bieler, N. Laman, H. M. van Driel, and A. L. Smirl, Appl. Phys. Lett. 86, 061102 (2005).
${ }^{21}$ C. L. Yang, H. T. He, Lu Ding, L. J. Cui, Y. P. Zeng, J. N. Wang, and W. K. Ge, Phys. Rev. Lett. 96, 186605 (2006).

${ }^{22}$ K. S. Cho, Y. F. Chen, Y. Q. Tang, and B. Shen, Appl. Phys. Lett. 90, 041909 (2007).

${ }^{23}$ M. Frazier, J. A. Waugh, J. J. Heremans, M. B. Santos, X. Liu, and G. A. Khodaparast, J. Appl. Phys. 106, 103513 (2009).

${ }^{24}$ S. D. Ganichev and W. Prettl, Intense Terahertz Excitation of Semiconductors (Oxford University Press, Oxford, UK, 2006).

${ }^{25}$ V. V. Bel'kov and S. D. Ganichev, Semicond. Sci. Technol. 23, 114003 (2008)

${ }^{26}$ V. V. Bel'kov, S. D. Ganichev, E. L. Ivchenko, S. A. Tarasenko, W. Weber, S. Giglberger, M. Olteanu, H.-P. Tranitz, S. N. Danilov, Petra Schneider, W. Wegscheider, D. Weiss, and W. Prettl, J. Phys.: Condens. Matter 17, 3405 (2005).

${ }^{27}$ S. D. Ganichev, E. L. Ivchenko, V. V. Bel'kov, S. A. Tarasenko, M. Sollinger, D. Weiss, W. Wegscheider, and W. Prettl, Nature (London) 417, 153 (2002).

${ }^{28}$ S. D. Ganichev, V. V. Bel'kov, S. A. Tarasenko, S. N. Danilov, S. Giglberger, Ch. Hoffmann, E. L. Ivchenko, D. Weiss, W. Wegscheider, Ch. Gerl, D. Schuh, J. Stahl, J. De Boeck, G. Borghs, and W. Prettl, Nat. Phys. 2, 609 (2006).

${ }^{29}$ V. Lechner, L. E. Golub, F. Lomakina, V. V. Bel'kov, P. Olbrich, S. Stachel, I. Caspers, M. Griesbeck, M. Kugler, M. J. Hirmer, T. Korn, C. Schüller, D. Schuh, W. Wegscheider, and S. D. Ganichev, Phys. Rev. B. 83, 155313 (2011).

${ }^{30}$ S. A. Tarasenko, Phys. Rev. B 77, 085328 (2008).

${ }^{31}$ S. A. Tarasenko, Phys. Rev. B 83, 035313 (2011).

${ }^{32}$ S. D. Ganichev, S. A. Emel'yanov, and I. D. Yaroshetskii, Pis'ma Zh. Eksp. Teor. Fiz. 35, 297 (1982) [JETP Lett. 35, 368 (1982)].

${ }^{33}$ S. D. Ganichev, W. Prettl, and P. G. Huggard, Phys. Rev. Lett. 71, 3882 (1993).

${ }^{34}$ S. D. Ganichev, I. N. Yassievich, W. Prettl, J. Diener, B. K. Meyer, and K. W. Benz, Phys. Rev. Lett. 75, 1590 (1995).

${ }^{35}$ P. Schneider, J. Kainz, S. D. Ganichev, V. V. Bel'kov, S. N. Danilov, M. M. Glazov, L. E. Golub, U. Rössler, W. Wegscheider, D. Weiss, D. Schuh, and W. Prettl, J. Appl. Phys. 96, 420 (2004).

${ }^{36}$ S. D. Ganichev, Phys. B (Amsterdam) 273-274, 737 (1999).

${ }^{37}$ S. D. Ganichev, Ya. V. Terent'ev, and I. D. Yaroshetskii, Pis'ma Zh. Tekh. Fiz. 11, 46 (1985) [Sov. Tech. Phys. Lett. 11, 20 (1985)].

${ }^{38}$ E. Ziemann, S. D. Ganichev, I. N. Yassievich, V. I. Perel, and W. Prettl, J. Appl. Phys. 87, 3843 (2000).

${ }^{39}$ In general, asymmetric zinc-blende structure based QWs grown on (001) exact oriented substrates belong to the $C_{2 v}$ symmetry group. A current at $B=0$ is not allowed for materials of this symmetry and in fact is not observed in 30-nm QW samples. Thus, the signal observed in 20-nm QW samples indicates a symmetry reduction of the investigated samples due to, e.g., misorientation of substrate or presence of a strain in the structures. We note that, although in the 20-nm QWs structures nonzero signal at $B_{x}=0$ is observed, its magnitude becomes smaller than that of magnetic-field-induced current even for rather low $B_{x} \approx 0.2 \mathrm{~T}$. 
${ }^{40}$ We note that our estimations show that for single-pulse excitation, the heating of lattice is negligibly small and does not contribute to the photoconductive signal, the result which is well known for such type of measurements (Ref. 24). We also note that comparison of photoconductive data with the temperature dependence of mobility can not be directly used to determine the electron temperature because, in the second case, rising of lattice temperature results in increase of number of phonons.
${ }^{41}$ S. D. Ganichev, S. N. Danilov, V. V. Bel'kov, S. Giglberger, S. A. Tarasenko, E. L. Ivchenko, D. Weiss, W. Jantsch, F. Schäffler, D. Gruber, and W. Prettl, Phys. Rev. B 75, 155317 (2007).

${ }^{42}$ B. E. A. Saleh and M. C. Teich, Fundamentals of Photonics (Wiley, New York, 2007).

${ }^{43}$ F. Perez, C. Aku-leh, D. Richards, B. Jusserand, L. C. Smith, D. Wolverson, and G. Karczewski, Phys. Rev. Lett. 99, 026403 (2007). 